\title{
FAM188B enhances cell survival via interaction with USP7
}

\author{
Eun-Seok Choi ${ }^{1,2}$, Hanna Lee', Jee Young Sung ${ }^{1}$, Chang-Hun Lee ${ }^{1}$, Hyonchol Jang $\mathbb{D}^{1}$, Kyung Tae Kim', \\ Yong-Nyun Kim', Hyoung-Pyo Kim² and Sung-Ho Goh ${ }^{1}$
}

\begin{abstract}
We have previously reported that FAM188B showed significant differential exon usage in cancers (NCBI GEO GSE30727), but the expression and function of FAM188B is not well characterized. In the present study, we explored the functions of FAM188B by a knockdown strategy, using siRNAs specific for FAM188B in colon cancer cell lines. FAM188B is a novel gene that encodes a protein that is evolutionarily conserved among mammals. Its mRNA has been found to be highly expressed in most solid tumors, including colorectal cancer. FAM188B knockdown induced cell growth inhibition due to an increase in apoptosis in colon cancer cell lines. Interestingly, siFAM188B treatment induced the upregulation and activation of p53, and consequently increased p53-regulated pro-apoptotic proteins, PUMA and BAX. Proteomic analysis of FAM188B immunocomplexes revealed p53 and USP7 as putative FAM188Binteracting proteins. Deletion of the putative USP7-binding motif in FAM188B reduced complex formation of FAM188B with USP7. It is noteworthy that FAM188B knockdown resulted in a decrease in overall ubiquitination in the p53 immunocomplexes, as well as p53 ubiquitination, because USP7 is involved in p53 deubiquitination. FAM188B knockdown inhibited both colony formation and anchorage-independent growth in vitro. In addition, FAM188B knockdown by siRNA reduced tumor growth in xenografted mice, with an increase in p53 proteins. Taken together, our data suggest that FAM188B is a putative oncogene that functions via interaction with USP7. Therefore, control of FAM188B could be a possible target to inhibit tumor growth.
\end{abstract}

\section{Introduction}

Colorectal cancer $(\mathrm{CRC})$ is the third most prevalent cancer worldwide and is a major contributor to cancer mortality ${ }^{1}$. CRC is heterogeneous disease, biologically classified into three major groups according to their molecular characteristics. The first is the chromosomal instable group, which accumulates mutations in specific oncogenes and tumor-suppressor genes. The second class is the microsatellite instability group, which leads to genetic hyper mutation, and the third is distinguished by CpG island methylation ${ }^{2}$. In addition, large-scale genomic studies have been conducted to advance our

\footnotetext{
Correspondence: S-H. Goh (andrea@ncc.re.kr)

${ }^{1}$ Research Institute, National Cancer Center, Goyang 10408, Republic of Korea ${ }^{2}$ Department of Environmental Medical Biology, Institute of Tropical Medicine, Yonsei University College of Medicine, Seoul 03722, Republic of Korea Edited by M. Diederich
}

understanding of CRC at a molecular level, including The Cancer Genome Atlas analysis of 276 colon cancer patients ${ }^{3}$. Many critical pathways contribute to the development of CRC, including APC, WNT, RAS-MAPK, PI3K, TGF- $\beta$, TP53, and DNA mismatch repair ${ }^{3}$. However, despite these efforts, there is still lack of detailed characterization for low to intermediate frequency mutations or novel candidates.

Programmed cell death inhibits the development of cancer naturally through apoptosis of abnormal cells, but cancer develops when this mechanism is disrupted ${ }^{4}$. Typically, when chromosomal abnormality occurs, the expression of tumor-suppressor P53 is increased, leading to apoptosis of the cells ${ }^{5}$. Regulation of p53 is controlled by various post-translational modifications. The ubiquitin-proteasome system (UPS) is the main pathway for controlling protein integrity, and is central to the 
regulation of many cellular functions, notably including cell survival and death ${ }^{6,7}$. Ubiquitination is a remarkably complex, specific, three-enzyme (E1-E2-E3) cascade that utilizes $2 \mathrm{E} 1,10 \mathrm{E} 2$, and hundreds of E3 ubiquitin ligases ${ }^{8}$. Deubiquitinases (DUB, ubiquitin isopeptidase) are UPS components that catalyze removal of an ubiquitin moiety from poly-ubiquitin chains ${ }^{6}$; the human genome encodes 98 DUB genes classified into six families ${ }^{9}$. Thus, the dynamic and combinatorial interactions between ubiquitination and deubiquitination set the threshold for apoptotic signaling ${ }^{10}$. For example, the E3 ubiquitin ligase MDM2 ubiquitinates the tumor-suppressor p53, and DUBs, such as ubiquitin-specific proteases USP2a, USP7, USP10, USP22, and USP42, are involved in regulating the stability of p53 and MDM2 by removing ubiquitin moieties $^{6,11-13}$. However, what determines whether p53 or MDM2 is the primary USP substrate is not known ${ }^{10,14}$.

A substantial proportion of genes (59\%) in the human genome are reported as "hypothetical" and are annotated as being of "unknown function" ${ }^{15}$. Hypothetical proteins are predicted from nucleic acid sequences and their existence has not been experimentally proven. Another feature of the hypothetical protein is that it has low identity compared to known proteins ${ }^{16}$. However, despite their hypothetical status, which can be an obstacle to investigations of their expression patterns and potential functions in cellular pathways, such genes are often expressed to varying degrees in disease and are therefore biomedically relevant ${ }^{17}$. Thus, excluding "unknown" or "hypothetical" genes from analyses of candidate targets removes the opportunity to explore unprecedented molecular mechanisms that may be involved in clinically significant pathological dysfunctions. Recently, a hypothetical protein, FAM63A, was characterized as a new DUB family member, and the analysis of evolutionarily conservation among human genomes identified FAM63B as a homolog, and listed FAM188A and FAM188B as evolutionarily distant members ${ }^{18}$.

In our previous study, FAM188B showed significant differential exon usage in cancers (NCBI GEO GSE30727) $)^{19}$, but the expression and function of FAM188B had not yet been characterized. However, public database search revealed FAM188B was differentially expressed in many cancer types, and CRC showed significantly elevated expression in tumor. Here, we provide the first data that FAM188B is a genuine gene that overexpressed in CRC. In addition, we show that it functions in sustaining cell survival in vitro, and regulates growth in vivo. Our analyses revealed FAM188B intervenes in p53 stability control through interactions with USP7.

\section{Results}

Upregulation of FAM188B expression in colorectal cancers

Our previous study indicated that $F A M 188 B$ has significant differential exon usage and differential expression in gastric cancer tissues ${ }^{19}$. To explore whether $F A M 188 B$ expression is altered in other cancers, we first searched messenger RNA (mRNA) expression profiles of cancer cell lines using the Cancer Cell Line Encyclopedia (CCLE) database $^{20}$. Most solid tumor cell lines, including CRC, showed a higher mRNA expression level of FAM188B than tumors from lymphoma, leukemia, and B-cell or chondrosarcoma (Fig. 1a). To verify FAM188B mRNA expression, we carried out quantitative real-time PCR (qRT-PCR) using various cancer and non-cancerous cell lines (Fig. 1b). FAM188B mRNA expression was detected to different levels in all cell lines tested, including CRC cell lines. To determine its endogenous protein expression, we generated a polyclonal antibody against FAM188B. Western blot analysis revealed that FAM188B protein was expressed in all cell lines (Fig. 1c). FAM188B protein levels appeared to be relatively low in human dermal fibroblasts (HDFs), which was correlated with low mRNA levels. As FAM188B expression at both mRNA and protein level were substantial in the CRC cell lines (HCT-116, SW620, and HT-29), we further explored public datasets regarding FAM188B expression in CRC patients. FAM188B mRNA expression was highly increased in multiple CRC datasets (TCGA colorectal 2, $p=2.48 \times 10^{-16}$; GSE20916, $p=6.59 \times 10^{-7}$; GSE20842, $p=2.68 \times 10^{-26}$ ) from Oncomine database ${ }^{21}$ (Fig. 1d).

\section{FAM188B downregulation leads to cell death in vitro}

To understand the function of $F A M 188 B$, we knocked down FAM188B expression using FAM188B-specific siRNA in the colon cancer cell lines HCT-116, HT-29, and SW620. Transfection of cells with siFAM188B decreased FAM188B mRNA expression from $24-96 \mathrm{~h}$ as determined by qRT-PCR (Fig. 2a and Supplementary Figures $3 \mathrm{a}$ and $4 \mathrm{a}$ ), whereas negative control (NC) siRNA had little effect on FAM188B expression. Correspondingly, FAM188B mRNA levels decreased. FAM188B protein levels were also reduced by siFAM188B, as determined by western blot (Fig. 2b, Supplementary Figures $3 \mathrm{~b}$ and $4 \mathrm{~b})$. To test the effects of FAM188B knockdown on cell growth, we employed two different siFAM188B RNAs (siFAM188B and siFAM188B-2). When FAM188B was knocked down by either siFAM188B or siFAM188B-2, HCT-116 cell growth decreased with time (Fig. 2c, Supplementary Figures 3c and 4c). A microscopic analysis of DAPI-stained cells also revealed that siFAM188B treatment increased brightly fluorescent and fragmented nuclei, which is indicative of apoptosis (Fig. 2d). As cell growth inhibition could occur either by cell-cycle arrest or cell death, we examined the effect of $F A M 188 B$ knockdown on cell cycle. Interestingly, the sub-G0/G1 population increased in the siFAM188Btreated cells, which corresponds to apoptotic cells (Fig. 2e). In addition, FAM188B knockdown increased 

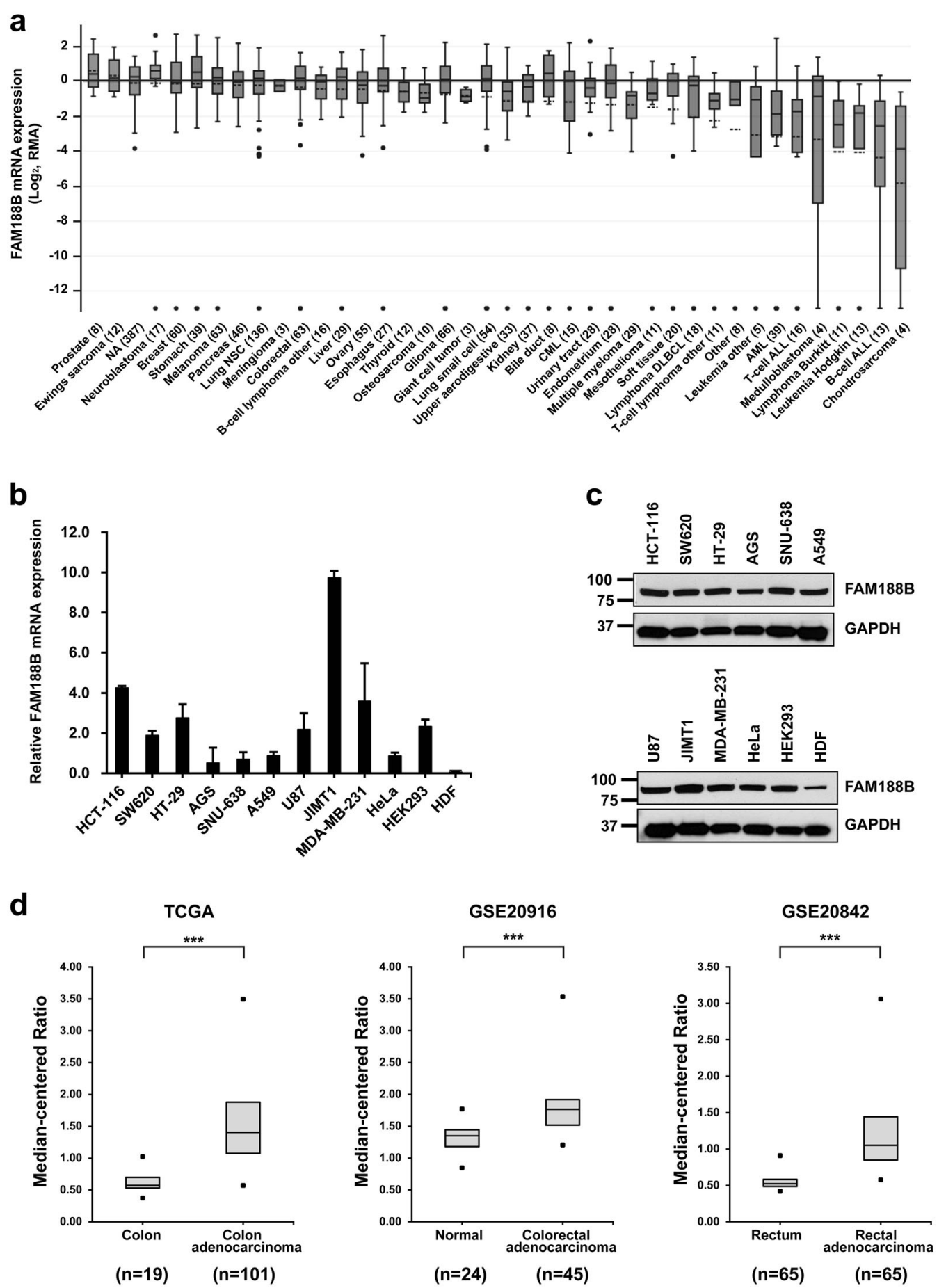

Fig. 1 FAM188B expression levels in public database and validation in cell lines. a FAM188B mRNA expression levels in CCLE database. Boxand-whisker plots show the distribution of mRNA expression for each cancer type. Line in the box indicates median and dashed line indicates mean. b FAM188B mRNA levels were measured in cancer cell lines (HCT-116, SW620, HT-29, AGS, SNU-638, A549, U87, JIMT1, MDA-MB-231, and HeLa) and non-cancer cell lines (HEK-293 and HDF). Error bar indicates S.D. c Proteins $(25 \mu \mathrm{g})$ from indicated cell lines were subjected to western blotting analysis using anti-FAM188B antibody. GAPDH was used as a loading control. Similar results were observed in three independent experiments. d FAM188B expression comparison between normal and tumor patient tissues using TCGA, GSE20916, and GSE20842 data set. Upper and lower dots indicate the maximum and minimum values. The middle line in the box indicates median $\left.{ }^{* * *} p<0.001\right)$ 
a

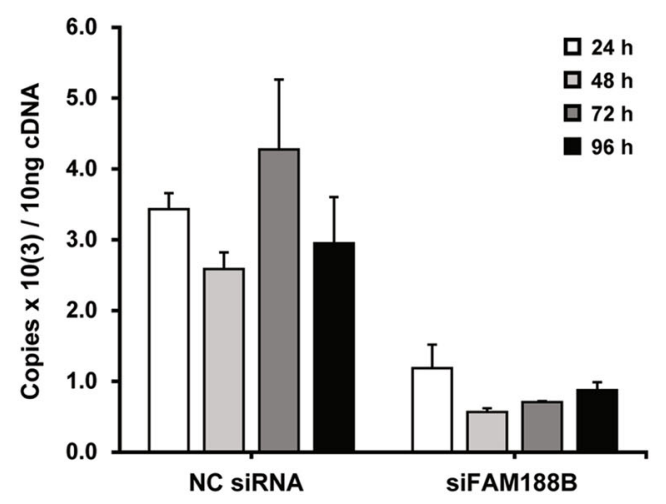

C

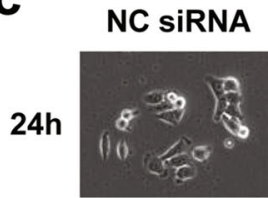

$48 \mathrm{~h}$

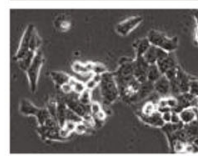

$72 \mathrm{~h}$
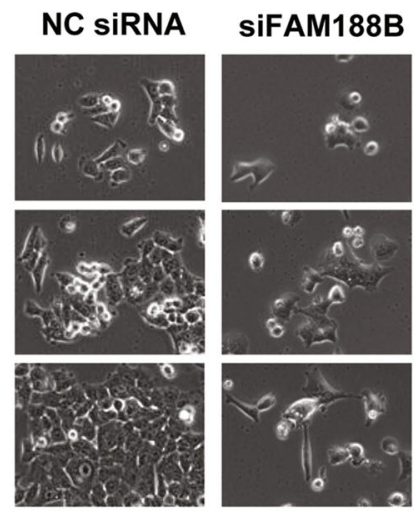

siFAM188B-2
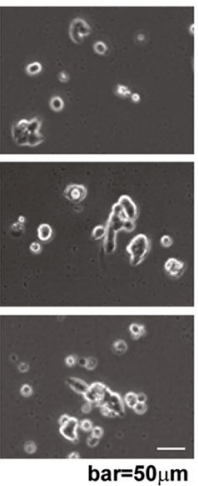

e NC SiRNA
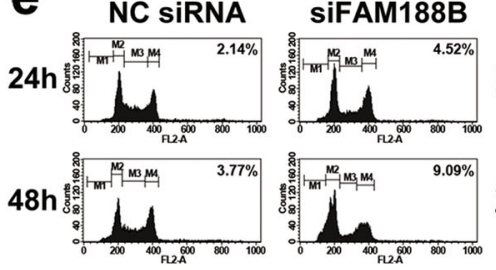

SiFAM188B-2
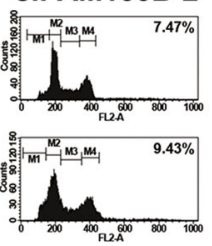

$72 \mathrm{~h}$
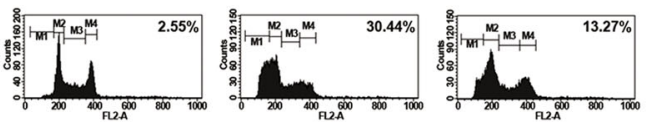

b
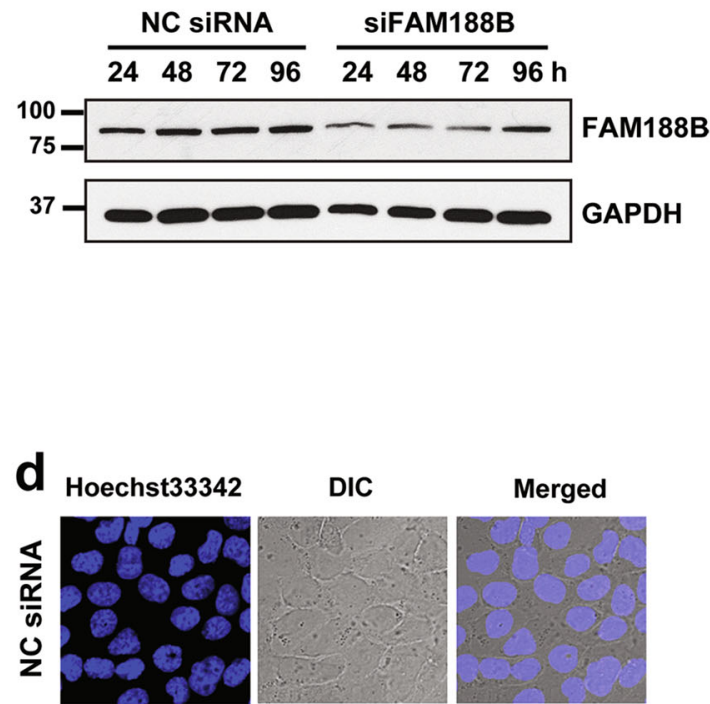

DIC
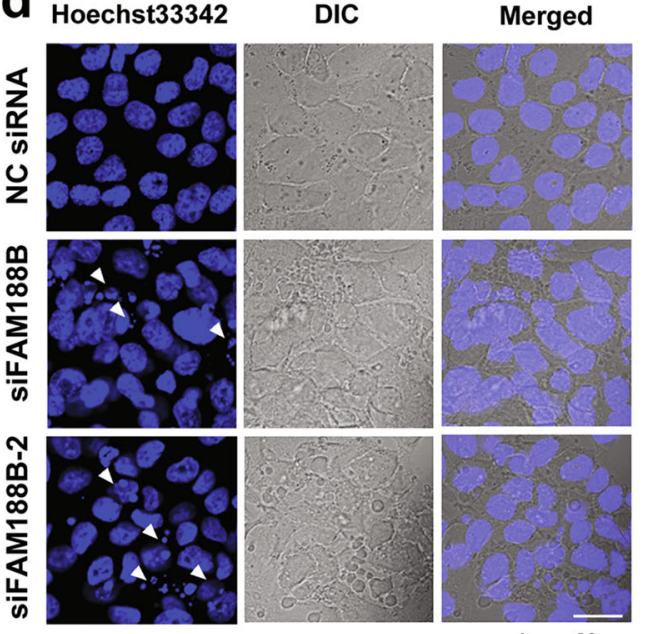

$72 \mathrm{~h}$

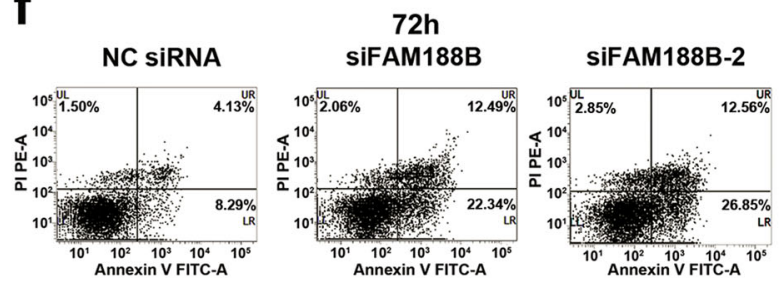

Fig. 2 Effects of FAM188B knockdown on cell death. $\mathbf{a}$, $\mathbf{b}$ HCT-116 cells were transfected with either NC siRNA or siFAM188B for indicated times. The mRNA levels and protein levels of FAM188B were measured by qRT-PCR (a) and western blotting (b), respectively. c-f HCT-116 cells were transfected with either NC siRNA, siFAM188B, or siFAM188B-2 and images for cell growth (c) and nuclei fragmentation (d) were taken at the indicated times. White arrow heads indicate nuclei fragmentation (scale bar $=20 \mu \mathrm{m})(\mathbf{d})$. Cells were also processed for cell-cycle analysis by flow cytometry; dead cell population: sub-G0/G1 is indicated in (\%) (e). For apoptosis analysis, after $72 \mathrm{~h}$ of siRNA transfection cells were stained with annexin V/PI, followed by flow cytometry (f) (PI-stained cells apoptosis population: UR and UL)

the population of annexin V/PI-stained cells (Fig. 2f). Apoptosis induced by siFAM188B was not limited to HCT-116 cells because compared with NC siRNA-treated cells, siFAM188B treatment also increased annexin V/PI-positive cells in other colon cancer cell lines, HT-29 and SW620 (Fig. 2f, Supplementary Figures 3d and 4d). All these data suggest that downregulation of FAM188B expression in colon cancer cells leads to apoptosis.
FAM188B silencing activates $\mathrm{p} 53$ and its downstream pathway

To investigate the mechanism of FAM188B involvement in cell death, we searched for potential cellular FAM188B-binding partners by liquid chromatography/ mass spectrometry (LC/MS)-MS (Fig. 3a). Mass peaks from MS searches against the database identified a total of 104 unique proteins bound to FAM188B. These proteins were analyzed using the DAVID server ${ }^{22}$ and String-DB ${ }^{23}$ 


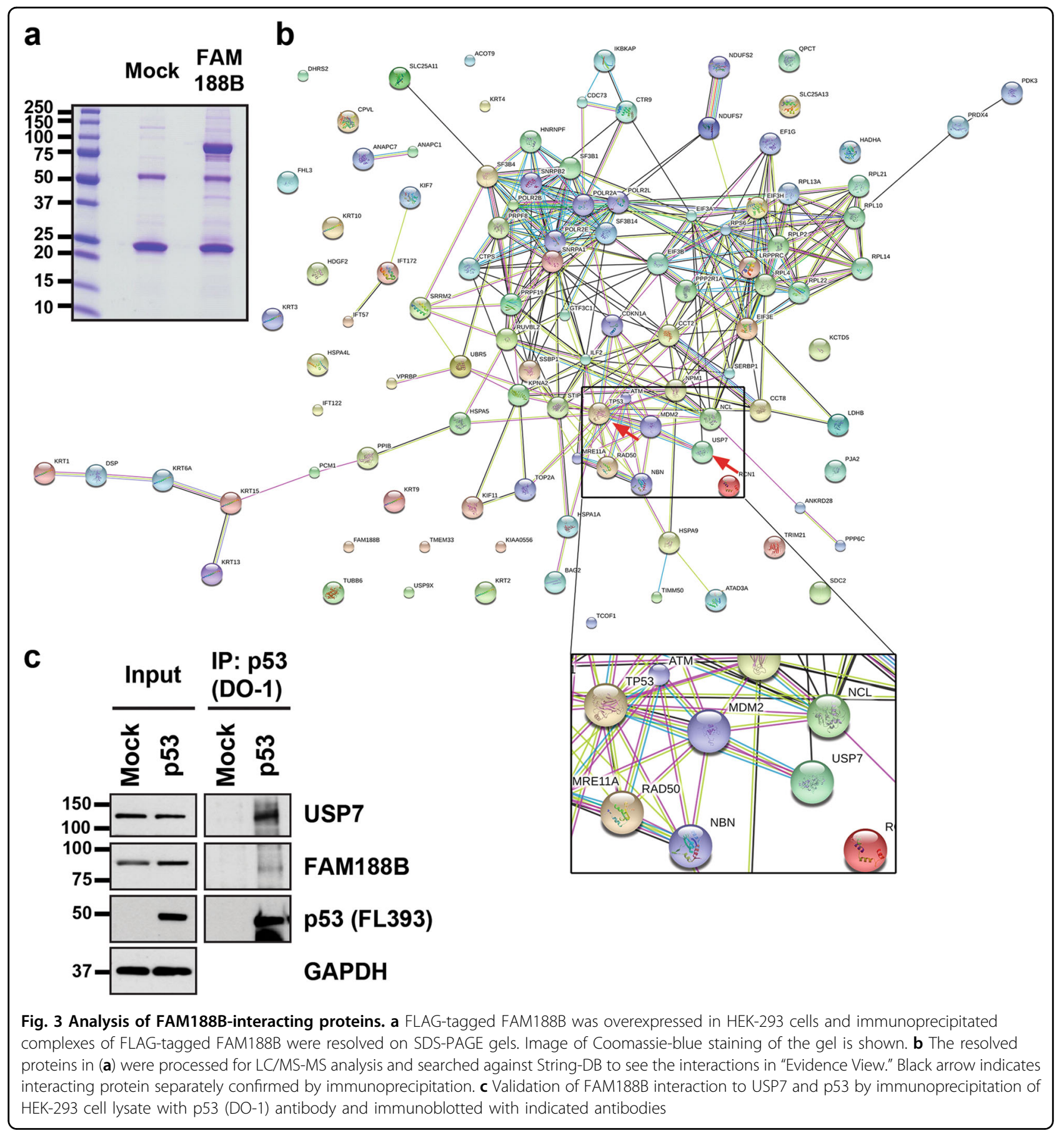

to cluster proteins according to their involvement in intracellular biological processes and evidence-based interacting groups, respectively. As expected, based on results obtained by FAM188B downregulation, many FAM188B-binding proteins were involved in cell-cycle regulation and apoptosis (Supplementary table 2). Notably, clusters on the interaction map of FAM188B-binding proteins revealed several clusters including the tumorsuppressor proteins p53 as well as USP7 (Fig. 3b, arrows).
Interestingly, when we immunoprecipitated p53 proteins, we could observe that FAM188B as well as USP7 were in the p53 immunocomplexes (Fig. 3c), indicating that FAM188B forms a complex with p53.

Because p53 is an important regulator for cell death control, we further examined a possible association of p53 in the siFAM188B-induced apoptosis in more detail. When cells were stained for p53, more p53 were localized in the nucleus in the siFAM188-treated cells than in the 


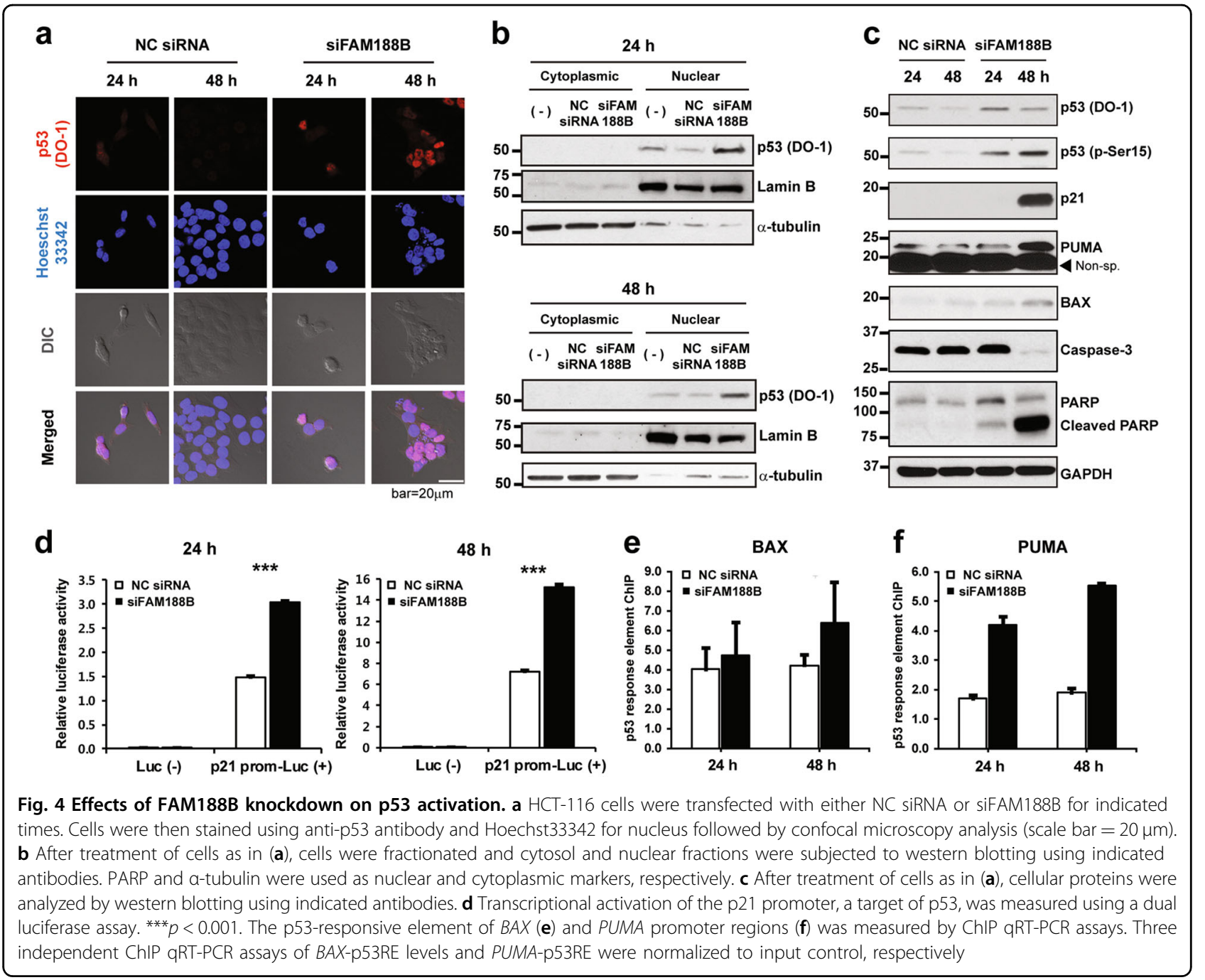

NC siRNA-treated cells (Fig. 4a). Consistent with these immunocytochemistry data, more p53 protein was detected in the nucleus fraction than in the cytosolic fraction when FAM188B was knocked down (Fig. 4b). Next, we tested whether p53 is activated in the FAM188B knocked-down cells by immunoblotting using antibodies that recognize Ser15-phosphorylated p53 (active form of p53) ${ }^{24}$. FAM188B knockdown increased p53 as well as Ser15-phosphorylated p53, and thus enhanced protein levels of p53-regulated genes, including p21, PUMA, and BAX, which are in the apoptosis pathway (Fig. 4c). To further test p53 activation transcriptionally, we performed promoter assay using p21 promoter-luciferase construct because $\mathrm{p} 21$ is known to be a p53 target gene ${ }^{25}$. siFAM188B treatment increased the transcriptional activity of p53 toward its downstream target p21 by approximately twofold (Fig. 4d). Moreover, chromatin immunoprecipitation (ChIP) assays using p53 antibodies showed increased binding of p53 to $B A X$ (Fig. 4e) and
PUMA promoters (Fig. 4f) in the siFAM188B-treated cells. These results suggest that FAM188B downregulation activates $\mathrm{p} 53$, and thus upregulates apoptosisrelated genes, including BAX, and PUMA, and leads to cell death.

FAM188B regulates p53 stability via interaction with USP7

To determine how FAM188B knockdown enhances p53 protein levels, we identified FAM188B-interacting proteins revealed by LC/MS-MS (Fig. 3b). As FAM188B silencing affects p53 levels, we tested whether USP7, a deubiquitinase, and p53-interacting proteins detected in LC/MS-MS experiments, can be detected as a FAM188Bbinding protein. In accordance with this, the online protein domain prediction site, "Eukaryotic Linear Motif resources" ${ }^{26}$, also predicted the presence of a USP7binding motifs in FAM188B (Fig. 5a and Supplementary Figure 1a). USP7 protein was detected in FAM188B immunoprecipitates in the cells with FAM188B 
overexpression (Fig. 5b), indicating a complex formation of FAM188B with USP7. To investigate which motif of FAM188B is responsible for its complex formation with USP7, either FLAG-tagged wild-type or mutant FAM188B with deletion of USP7-binding motif-1 (AUSP7-1) or USP7-binding motif-2 ( $\triangle$ USP7-2) were overexpressed in HCT-116 cells, followed by immunoprecipitation of exogenous FAM188B using anti-FLAG antibodies. USP7 was consistently found in the wild-type FAM188B immunocomplexes but USP7 levels decreased in the mutant FAM188B immunocomplexes ( $\triangle$ USP7-2), suggesting that USP7-2 motifs of FAM188B are important for its complex formation with USP7 (Fig. 5c). Next, to examine whether USP7 interacts with p53, we performed a co-IP assay after etoposide treatment to increase p53 expression. Etoposide treatment resulted in an increase in p53 and a decrease in FAM188B. P53 was found in the USP7 immunocomplexes and this complex formation increased with etoposide treatment (Fig. 5d). Next, we tested whether FAM188B levels affect complex formation of USP7 and p53. A basal level of USP7 was detected in the p53 immunocomplexes. However, interestingly, this complex formation appeared increased in the siFAM188B-treated cells after $24 \mathrm{~h}$, and it became weaker at $48 \mathrm{~h}$ although the endogenous USP7 protein expression was not changed by FAM188B silencing (Fig. 5e).

To test whether FAM188B is involved in p53 deubiquitination, we examined the change in the level of ubiquitinated p53 after FAM188B knockdown in the cells expressing HA-tagged ubiquitin. Compared with NC siRNA-treated cells, less p53 was detected in the HAimmunoprecipitated complexes from siFAM188B-treated cells (Fig. 5f). In addition, the level of HA-tagged ubiquitin decreased in the p53 immunocomplexes from siFAM188B-treated cells compared with those of NC siRNA-treated cells. A lower level of endogenous ubiquitinated p53 was also detected in the p53 immunocomplexes from siFAM188B-treated cells than those of NC siRNA-treated cells (Fig. 5g).

\section{FAM188B knockdown inhibits tumor growth in vivo}

To further investigate the effect of FAM188B downregulation on tumor growth, we established a shFAM188B-inducibile HCT-116 stable cell line. Doxycycline induced $F A M 188 B$ short hairpin RNA (shRNA), which led to FAM188B downregulation (Fig. 6a). FAM188B shRNA expression reduced the number of colonies (Fig. 6b). Next, we examined the effects of FAM188B knockdown on anchorage-independent colony formation by soft agar assay. When HCT-116 cells were treated with siFAM188B, anchorage-independent colony formation was significantly decreased (Fig. 6c). These data indicated that FAM188B might have an oncogenic function. To test the effect of FAM188B silencing on tumor growth in vivo, HCT-116 cells were xenografted into $\mathrm{BALB} / \mathrm{c}$ nude mice, and $F A M 188 B$ small interfering RNA (siRNA) was delivered by electroporation. Tumor volume was regularly measured after treatment with $F A M 188 B$ siRNA. The growth of siFAM188B-treated tumors was significantly reduced from the first week compared to that of NC siRNA-treated tumors (Fig. 6d). When the tumors were removed from the killed mice, siFAM188B-treated tumors were smaller than the NC siRNA-treated tumors (Fig. 6d). To verify the FAM188B knockdown in the xenografted tumors, tumor tissues were processed for immunostaining using anti-FAM188B antibodies. Immunohistochemistry analysis showed that FAM188B protein levels reduced, while p53 protein levels increased, in the siFAM188B-treated tumors (Fig. 6e). These data indicate that FAM188B expression is important for tumor growth in vitro and in vivo. Taken altogether, our data indicate that FAM188B has a critical oncogenic effect, possibly via enhancing p53 ubiquitination and thus p53 downregulation. Therefore, targeting FAM188B could be a good strategy to control tumor growth.

\section{Discussion}

Cancer progression is the result of various biological processes, and may also involve unexplored functions of novel/hypothetical genes, and/or variants of known genes. Despite the tremendous effort devoted to analyze available genomic information, as much as 59\% of human genes were annotated as "hypothetical" when the human genome was first reported ${ }^{15,27}$, and $24-31 \%$ of entrez/ ensemble database entries are currently annotated as "uncharacterized"17. Our previous study, profiling exon usage in gastric cancer, identified $F A M 188 B$ as a gene that was significantly alternatively spliced between gastric cancer and adjacent normal tissue. Until now, FAM188B was annotated as a hypothetical gene for which the only evidence for function was its transcript; no confirmation of its expression at the protein level had yet been provided.

Before we characterized the function of FAM188B, we first analyzed the evolutionary conservation of the protein sequences among species, because a biologically important gene would be preserved in the genome, even if its function is not known. We obtained 15 orthologous protein sequences to FAM188B from bony fish to chimpanzee from GenBank and aligned these sequences with human FAM188B (Supplementary Figure 1a) and compared the evolutionary distance among them (Supplementary Figure 1b). All of the homologs showed high similarity, and the sequence similarity of DUF4205 (domain of unknown function: IPR025257) ${ }^{28}$ among mammalian FAM188B homologs was $>76 \%$ (Supplementary Figure 1a). According to the cladogram, human FAM188B was closely related with the other orthologues 
a

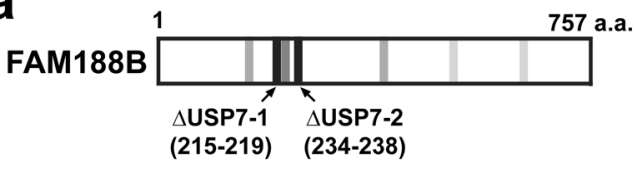

b

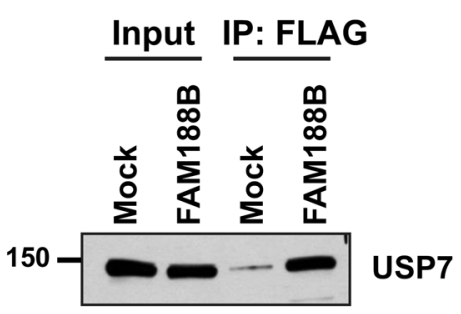

d

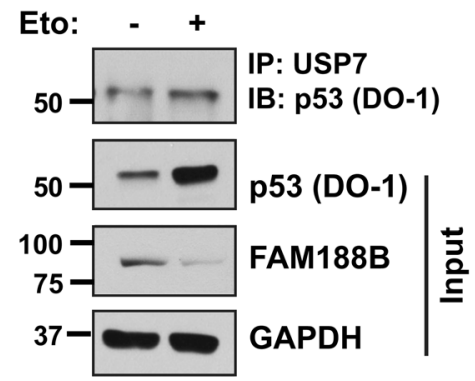

C FAM188B

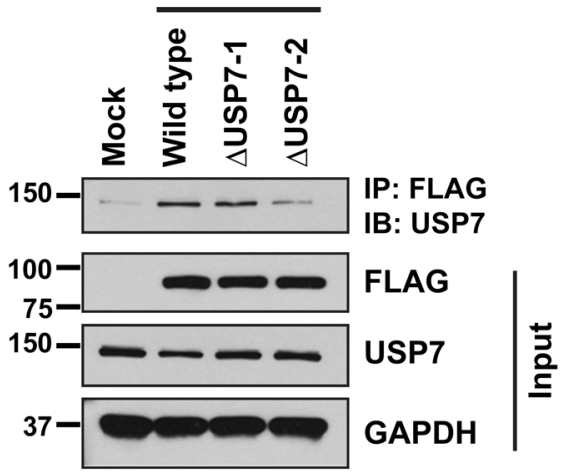

$\begin{array}{ccc}\text { N } & \text { NC } & \text { SiFAM } \\ \text { SiRNA } & \text { 188B }\end{array}$ SiRNA 188B $24 \quad 48 \quad 24 \quad 48 \mathrm{~h}$

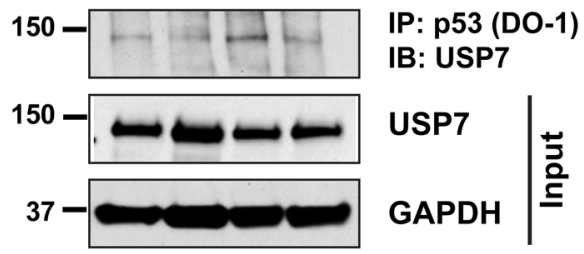

f

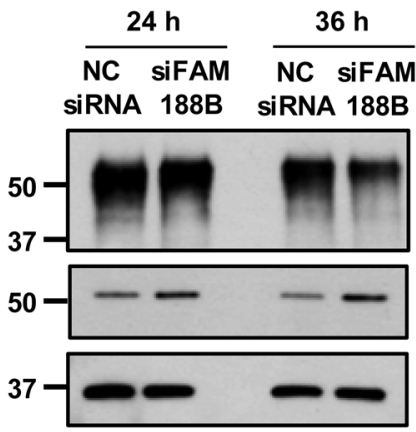

IP: HA

IB: p53 (DO-1)

IB: $\mathrm{p} 53$ (DO-1)

IB: GAPDH

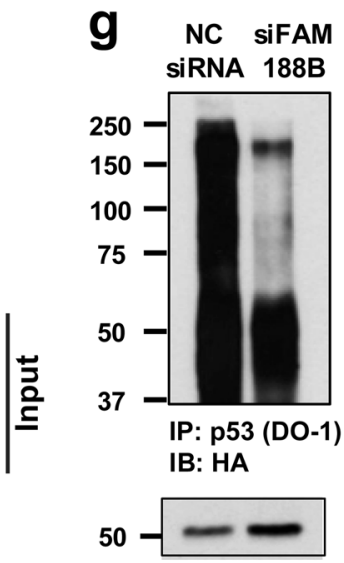

IB: p53 (DO-1)

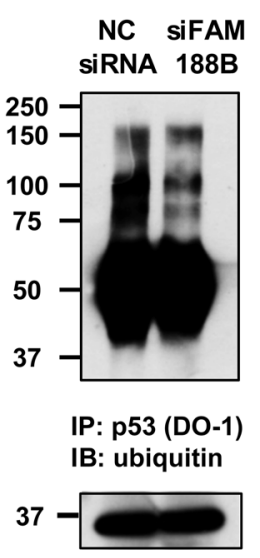

IB: GAPDH

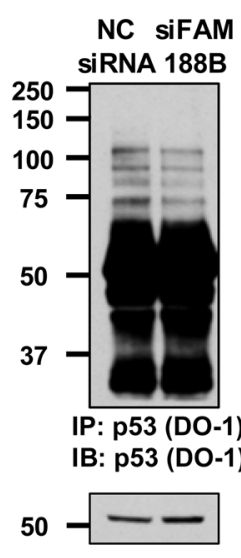

IB: p53 (DO-1)

Fig. 5 USP7 interacts with FAM188B and knockdown of FAM188B regulates $\mathrm{p} 53$ deubiquitination. a Predicted position of seven USP7-binding motifs were indicated on FAM188B protein. $\triangle$ USP7-1 and -2 are the positions of deletion mutants to analyze FAM188B-USP7 interaction. b Coimmunoprecipitation of overexpressed FAM188B and USP7 in HCT-116 cell. c Verification of the interaction between FAM188B and USP7 using overexpression of wild-type and the predicted USP7-binding motif deleted FAM188B constructs. $\mathbf{d}$ Lysates from HCT-116 cells treated with or without etoposide $(5 \mu \mathrm{M})$ were immunoprecipitated and immunoblotted with antibodies shown in the figure. e FAM188B silencing increased the interaction between USP7 and p53. Cell lysates from HCT-116 cells treated with NC siRNA or siFAM188B for 24 and $48 \mathrm{~h}$ were immunoprecipitated with anti-p53 antibody and immunoblotted with anti-USP7 antibody. $\mathbf{f}, \mathbf{g}$ Knockdown of FAM188B reduces ubiquitinated p53. $\mathbf{f}$ HA-tagged ubiquitin (5 $\mu \mathrm{g})$ was transfected to HCT-116. Cell lysates from HCT-116 treated with NC siRNA or siFAM188B were immunoprecipitated by anti-HA antibody and immunoblotted with indicated antibodies. $\mathbf{g}$ Cell lysates from HCT-116 co-transfected with HA-tagged ubiquitin and NC siRNA or siFAM188B were immunoprecipitated by anti-p53 (DO-1) antibody and immunoblotted with indicated antibodies 


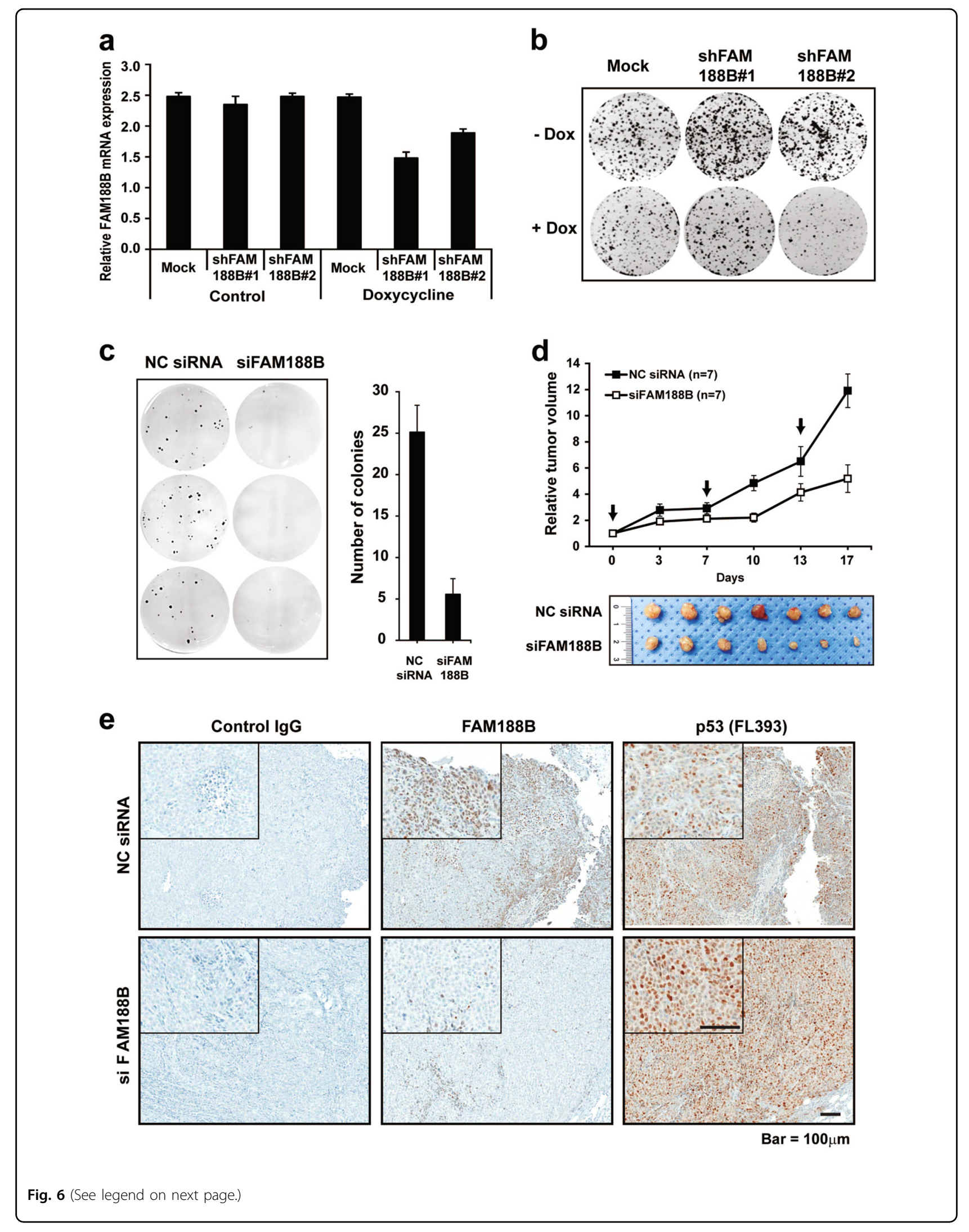




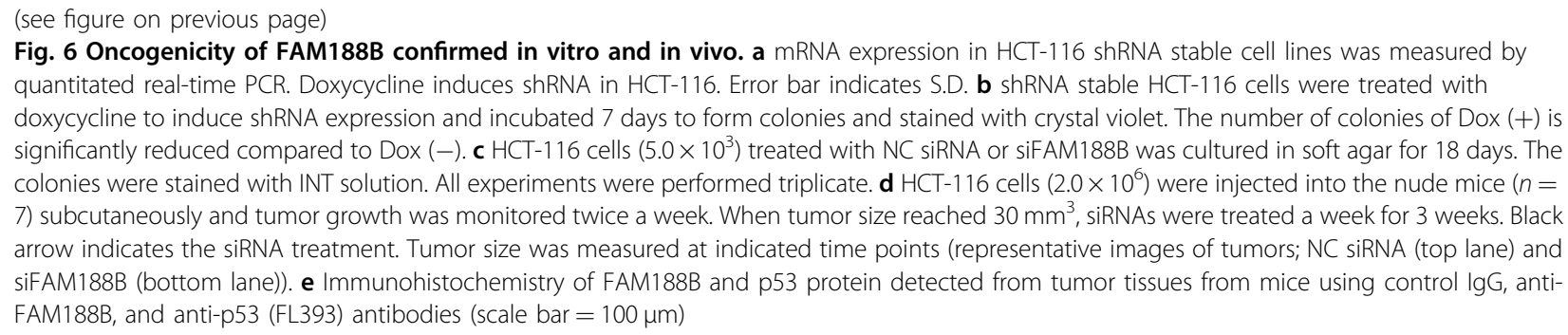

from primates such as Pan troglodytes (chimpanzee), Pongo abelii (orangutan), but far from amphibian Xenopus tropicalis (Supplementary Figure 1b). These results suggest that FAM188B might be conserved through the evolutionary lineage of the vertebrates for a biologically indispensable function.

We first determined whether this gene is a protein-coding gene or a non-coding gene. The mature mRNA sequence of FAM188B from the colon cancer cell line HCT-116 showed a poly-A signal sequence in its $3^{\prime}$ UTR (data not shown), suggesting that this gene could indeed encode a protein. This evidence led us to generate a rabbit polyclonal antibody to detect the FAM188B protein. Using the results of anti-FAM188B-specific antibody, we could detect endogenously or overexpressed FAM188B protein by immunoblotting and immunohistochemistry (Supplementary Figure 2). Furthermore, as revealed by transcriptome profiles of the public databases including the CCLE, Oncomine, and NCBI $\mathrm{GEO}^{29}$, the expression of FAM188B was significantly enhanced in tumors at the mRNA level. These results suggest that $F A M 188 B$ has important housekeeping functions in maintaining cell viability.

Having obtained evidence of endogenous expression, we explored the functional implications of elevated $F A M 188 B$ expression in tumors by suppressing translation of its mRNA using a siRNA approach. This loss-offunction study showed that knockdown of $F A M 188 B$ resulted in a significant increase in apoptotic cellular phenotypes, including accumulation of a sub-G0/G1 cellcycle population, fragmented nuclei, and an increase in the dead cell population. These phenotypic changes were also observed in other cancer type cell (AGS, a gastric cancer cell; Supplementary Figure 5), thus providing strong evidence of the potential role of $F A M 188 B$ as an oncogene or regulator of oncogenic pathways. However, identifying genuine interacting partners-a key to the known world of biological pathways-is crucial to understanding the working mechanisms of this unknown protein. The catalog of FAM188B-interacting proteins was defined through immunoprecipitation of FAM188B followed by LC/MS-MS analysis. A clustering of these proteins according to their gene ontology (GO) biological process showed that the major clusters were "protein translation" and "cell death/apoptosis," followed by "chromatin regulation" and "RNA-binding proteins" (Supplementary Table 2). Among these biological processes, cell death and protein degradation categories were of interest, because knockdown experiments suggested that this protein might be involved in regulating cell survival. Thus, we verified the FAM188B-interacting proteins identified by mass spectrometry using coimmunoprecipitation, and then proceeded to characterize FAM188B functions related to cell death. Immunoprecipitation using an anti-p53 (DO-1) antibody confirmed the interaction of FAM188B with p53 (Fig. 3c). Intriguingly, $\mathrm{GO}$ analyses categorized several proteins into an "ubiquitin-dependent protein" group; among them was USP7 (also known as HAUSP), which clustered with p53 as a FAM188B-interacting protein (Fig. 3b). USP7 is known to stabilize p53 by deubiquitinating it, as well as its inhibitor MDM2 ${ }^{30,31}$. As such, USP7 has come to be considered a therapeutic target in many cancers ${ }^{6,13}$. In breast cancer, TSPYL5 was reported to reduce p53 levels through physical interactions with $\mathrm{USP}^{32}$. In addition to USP7, USP2a and USP10 are also involved in regulating $\mathrm{p} 53$ ubiquitination in different contexts ${ }^{6}$; thus, evidence supporting USP7 as the major p53 modulator is inconclusive. In our study, however, the amount of FAM188B-bound USP7 dramatically increased upon FAM188B overexpression in HCT-116 cells. Conversely, FAM188B downregulation increased unbound USP7 and this free USP7 may decrease the level of ubiquitinated p53. The fact that USP7 plays a pivotal role in deubiquitinating both p53 and MDM2, as noted above, seemingly undermines the interpretation that USP7 is a p53 stabilizer. This may be the reason why the decrease of ubiquitinated p53 in siFAM188B-treated cells was not extreme (Fig. 5g). However, the shorter half-life of MDM2 compared to p53, as a result of MDM2 selfubiquitination $^{14,33}$, may ultimately lead to cell death through accumulated p53. If a mediator exists that could be the answer to the questions "What determines whether p53 or MDM2 is the primary USP7 substrate?"10 and "What determines how USP7 is regulated?"14, FAM188B might be that mediator. In tumor cells, high levels of FAM188B would be predicted to function by 
eliminating p53 and keeping it at a low level to allow tumor cells to grow. Silencing of USP7 decreased the p53 level and increased ubiquitinated-p53. However, double silencing of USP7 and FAM188B restored the p53 level (Supplementary Figure 6a). Therefore, elevated expression of FAM188B in tumor tissues suggests that FAM188B would inhibit p53 activation and thereby provide tolerance against stressful conditions (Supplementary Figure $6 \mathrm{~b}$ ). On the other hand, many tumors have mutations in or deletion of p53 gene, therefore, the effect of elevated FAM188B may not be exclusive on the tumorigenesis in such tumors.

The most intriguing result in this study was confirming the tumorigenicity of FAM188B. The colony forming ability of FAM188B was significantly abolished by silencing its expression in vitro. Interestingly, in soft agar colony formation assays, a golden standard to determine cellular transformation and tumorigenicity ${ }^{34}$, FAM188B silencing showed dramatic reduction of anchorageindependent colony growth (Fig. 6b). Moreover, in vivo tumor growth assays also revealed that FAM188B led to a dramatic difference in tumor masses of xenografted HCT116 in BALB/c nude mice. Accordingly immunohistochemical analyses revealed that FAM188B is a vital gene for proliferation or survival. Although additional studies with human tissue are also required to assess feasibility, these results suggest that FAM188B overexpression could be used as a predictive biomarker for cancer diagnosis, and suggests that inhibiting FAM188B activity could be utilized as the putative target of cancer progression. Although further studies are required to reveal how FAM188B expression is regulated, our findings clearly support the conclusion that FAM188B is an important regulator of p53 stability in growing cells.

In this study, the hypothetical protein FAM188B was revealed to be a genuine protein whose expression is significantly elevated in colon cancer cell lines. On the basis of FAM188B loss-of-function analyses, we suggest that FAM188B functions to sustain cell viability by decreasing p53 activation, through inhibition of the deubiquitinase USP7. This provides significant insight into the importance of FAM188B in sustaining cell survival, as well its use as a potential target in cancer therapy. Further research on the details of FAM188B regulatory mechanisms, as well conditions with p53 mutations, will increase our understanding of FAM188B as a potential therapeutic target.

\section{Materials and methods}

\section{Datasets from the public databases}

Datasets were obtained from Cancer Cell Line Encyclopedia (CCLE) database (https://portals.broadinstitute. org/ccle $)^{20}$ for cancer cell lines. Datasets of colorectal adenocarcinoma with $>2 \times$ of expression difference with $p$ - value $<0.001$ between normal and tumor tissues were obtained from Oncomine (https://www.oncomine.org/) ${ }^{21}$.

\section{Cell lines}

The human cell lines used in these studies (HCT-116, SW620, HT-29, AGS, SNU-638, A549, U87, JIMT1, MDA-MB-231, HeLa, HEK293, and HDF) were obtained from American Type Culture Collection (Manassas, VA, USA) or Korean Cell Line Bank (Seoul, Korea). All the cells were cultured with designated media (Corning, Manassas, VA, USA) supplemented with $10 \%$ fetal bovine serum (Corning) and $1 \times$ penicillin streptomycin (Invitrogen, Carlsbad, CA, USA) at $37^{\circ} \mathrm{C}$ containing $5 \% \mathrm{CO}_{2}$.

\section{Reverse-transcription polymerase chain reaction and quantitative RT-PCR}

First-strand complementary DNA synthesis and qRTPCR were performed as our previous report using a LC480 real-time PCR machine (Roche, Switzerland) ${ }^{35}$. Primers spanning two consecutive exons (Supplementary Table 1) were designed using Primer3 software (http:// frodo.wi.mit.edu/primer3/) ${ }^{36}$.

\section{Antibodies for immunoblot and immunocytochemistry}

For the detection of FAM188B, we generate polyclonal antiserum using FAM188B protein C-terminus peptides (722-738 a.a: TISEDTDNDLVPPLELC) as antigen (AbFrontier, Seoul, Korea) (Supplementary Figure 2). The affinity-purified serum was applied to immunoblots at 1:1000 dilutions in 5\% skim milk/1× Tris-buffered saline (TBS). The following antibodies were used to detect other proteins in this study: FLAG M2 and $\beta$-actin from SigmaAldrich (St. Louis, MO, USA); PUMA, USP7, p21, BAX and PARP from Cell Signaling Technologies (Danvers, MA, USA), and p53 (DO-1), p53 (FL393), phospho-p53 (ser15), and $\alpha$-tubulin from Santa Cruz Biotechnology (Dallas, TX, USA), and GFP (JL-8) from Clontech (Mountain view, CA, USA).

\section{Analyses of FAM188B expression knockdown}

FAM188B expression was silenced by transfecting with FAM188B-specific siFAM188B (Qiagen, Germany) targeting the sequence 5'-CTGACCATTGACACCACCAA-3', Allstar NC siRNA (Qiagen) at $5 \mathrm{nM}$ using Lipofectamine RNAiMAX (Thermo Fisher Scientific, San Jose, CA, USA).

Cell cycle was analyzed by flow cytometry by staining with propidium iodide (Sigma-Aldrich). Nuclear fragmentation in siRNA-treated cells stained with Hoechst33342 (Sigma-Aldrich) was observed by confocal microscopy at $\times 400$ magnification. Annexin $\mathrm{V}$ assays for the detection of apoptotic populations was carried out using a BD FITC annexin V apoptosis detection kit I (BD Pharmingen, San Jose, CA, USA) according to the manufacturer's protocol. 


\section{Colony forming assay and soft agar assay}

Doxycycline inducible HCT-116 shRNA (shFAM 188B\#1: CTTTGGAAATACGGCTAACAA, \#2: CAGAT ACTTTCTGGATCACTT) stable cell lines $\left(2 \times 10^{3}\right)$ seeded in six-well plates and $1 \mu \mathrm{g} / \mathrm{ml}$ of doxycycline (SigmaAldrich) was treated for 1 week. Colony formation was analyzed by fixing with $4 \%$ formaldehyde and staining with crystal violet solution (Sigma-Aldrich).

Onto the base agar $(0.8 \%)$ dispensed into the wells, siRNA-treated HCT-116 cells were seeded with top agar $(0.3 \%)$. Cells were cultured for 3 weeks with medium change every 4-5 days, and stained with INT solution.

\section{Proteomic analysis and profiling of interacting partners}

FAM188B-interacting proteins from HEK-293 cells transfected with pFLAG-FAM188B plasmid were immunoprecipitated with anti-FLAG-M2 agarose affinity gel (Sigma-Aldrich). Analyses were performed using an LTQ $\mathrm{XL}$ linear ion trap mass spectrometer (MS) system (Thermo Fisher Scientific). All MS/MS samples were analyzed using Proteome Discover software (version v.1.4; Thermo Fisher Scientific), set up to search the Uniprot database and IPI human database.

\section{Dual luciferase assay}

HCT-116 cells were transfected with NC siRNA or siFAM188B with pGL2-p21-luc (Addgene, Cambridge, MA, USA) and pGL4.70hRluc (Promega, Madison, WI, USA) plasmids. After 24 and $48 \mathrm{~h}$, cells were collected, and dual luciferase assays were performed according to the manufacturer's protocol (Promega) and measured using a Victor ${ }^{3}$ reader (Perkin Elmer).

\section{Chromatin immunoprecipitation assay}

Collected nuclei from fixed cells $(1 \times \mathrm{PBS} / 1 \%$ formaldehyde) were sonicated for $7.5 \mathrm{~min}(0.5 \mathrm{~min}$ on, $1 \mathrm{~min}$ off cycles) using a Bioruptor (Diagenode, Denville, NJ, USA). Fragmented genomic DNA was immunoprecipitated with anti-p53 antibody (DO-1), and purified by phenol/chloroform/isoamyl alcohol extraction. Amplification of $B A X$ and PUMA genes was carried out using SYBR green master mix with p53-RE primers ${ }^{37}$ using LC480 PCR machine (Roche).

\section{p53 ubiquitination assay}

HCT-116 cells were co-transfected with HA-Ubi plasmid and NC siRNA or siFAM188B. MG132 treated for $4 \mathrm{~h}$ before collection. One milligram of cell lysates were immunoprecipitated with anti-p53 (DO-1) antibody and immunoblotted with anti-HA antibody and vice versa. For the input control, $20 \mu \mathrm{g}$ of lysates were loaded and immunoblotted with p53 (DO-1), ubiquitin, HA, and GAPDH antibodies.

\section{In vivo analysis of $F A M 188 B$ silencing}

HCT-116 $\left(2.0 \times 10^{6}\right)$ cells were subcutaneously injected in 6-week-old male BALB/c nude mice (CAnN.CgFoxn1nu/CrljOri, Orient Bio, Korea). When the tumor size reached $30 \mathrm{~mm}^{3}$, Allstars NC siRNA or siFAM188B were injected into tumor via electroporation using NEPA21 Super Electroporator TypeII (Nepa Gene Co., Chiba, Japan) every 7 days for up to 3 weeks. Tumor volume was calculated by the modified ellipsoidal formula (length $\times$ width $\times$ width) $/ 2$. The experiment procedure and protocol was approved by Institutional Animal Care and Use Committee in National Cancer Center of Korea (NCC-16-231).

\section{Immunohistochemical staining}

Formaldehyde-fixed paraffin-embedded tissue section was used for immunohistochemical staining performed with Discovery XT (Ventana Medical Systems, Tucson, AZ, USA) using anti-FAM188B antibody (Atlas Antibodies AB, Stockholm, Sweden). Parallel sections incubated with normal IgG antibody instead of primary antibodies were used as NCs.

\section{Statistical analysis}

The statistical significance of differences between groups was determined using $\chi^{2}$-test and Student's $t$-test. $p$-values $<0.05$ were considered statistically significant.

\section{Acknowledgements}

This study was supported by Intramural Research Grant of National Cancer Center, Korea $(1810081,1611142)$ and National Research Foundation of Korea grant funded by the Ministry of Education of Korea (NRF-2013R1A1A2006712).

Conflict of interest

The authors declare that they have no conflict of interest.

\section{Publisher's note}

Springer Nature remains neutral with regard to jurisdictional claims in published maps and institutional affiliations.

Supplementary Information accompanies this paper at https://doi.org/ 10.1038/s41419-018-0650-6.

Received: 21 December 2017 Revised: 17 April 2018 Accepted: 2 May 2018 Published online: 24 May 2018

\section{References}

1. Torre, L. A. et al. Global cancer statistics, 2012. CA Cancer J. Clin. 65, 87-108 (2015).

2. Bogaert, J. \& Prenen, H. Molecular genetics of colorectal cancer. Ann. Gastroenterol. 27, 9-14 (2014).

3. Cancer Genome Atlas Network. Comprehensive molecular characterization of human colon and rectal cancer. Nature 487, 330-337 (2012).

4. Wong, R. S. Apoptosis in cancer: from pathogenesis to treatment. J. Exp. Clin Cancer Res. 30, 87 (2011).

5. Junttila, M. R. \& Evan, G. I. p53--a Jack of all trades but master of none. Nat. Rev. Cancer 9, 821-829 (2009). 
6. D'Arcy, P., Wang, X. \& Linder, S. Deubiquitinase inhibition as a cancer therapeutic strategy. Pharmacol. Ther. 147, 32-54 (2015).

7. Lopez-Otin, C. \& Hunter, T. The regulatory crosstalk between kinases and proteases in cancer. Nat. Rev. Cancer 10, 278-292 (2010).

8. Deshaies, R. J. \& Joazeiro, C. A. RING domain E3 ubiquitin ligases. Annu. Rev. Biochem. 78, 399-434 (2009).

9. Fraile, J. M., Quesada, V., Rodriguez, D., Freije, J. M. \& Lopez-Otin, C. Deubiquitinases in cancer: new functions and therapeutic options. Oncogene 31, 2373-2388 (2012).

10. Vucic, D., Dixit, V. M. \& Wertz, I. E. Ubiquitylation in apoptosis: a posttranslational modification at the edge of life and death. Nat. Rev. Mol. Cell Biol. 12, 439-452 (2011).

11. Li, M., Brooks, C. L., Kon, N. \& Gu, W. A dynamic role of HAUSP in the p53Mdm2 pathway. Mol. Cell 13, 879-886 (2004).

12. Meulmeester, E. et al. Loss of HAUSP-mediated deubiquitination contributes to DNA damage-induced destabilization of $\mathrm{Hdmx}$ and $\mathrm{Hdm} 2$. Mol. Cell 18, 565-576 (2005).

13. Pfoh, R., Lacdao, I. K. \& Saridakis, V. Deubiquitinases and the new therapeutic opportunities offered to cancer. Endocr. Relat. Cancer 22, T35-T54 (2015).

14. Brooks, C. L. \& Gu, W. p53 ubiquitination: Mdm2 and beyond. Mol. Cell 21 307-315 (2006).

15. Venter, J. C. et al. The sequence of the human genome. Science $\mathbf{2 9 1}$ 1304-1351 (2001).

16. Lubec, G., Afjehi-Sadat, L., Yang, J. W. \& John, J. P. Searching for hypothetical proteins: theory and practice based upon original data and literature. Prog. Neurobiol. 77, 90-127 (2005).

17. Pawlowski, K. Uncharacterized/hypothetical proteins in biomedical 'omics' experiments: is novelty being swept under the carpet? Brief Funct. Genomic. Proteomic. 7, 283-290 (2008).

18. Abdul Rehman, S. A. et al. MINDY-1 is a member of an evolutionarily conserved and structurally distinct new family of deubiquitinating enzymes. Mol. Cell 63, 146-155 (2016).

19. Nam, S. et al. Differential gene expression pattern in early gastric cancer by an integrative systematic approach. Int. J. Oncol. 41, 1675-1682 (2012).

20. Barretina, J. et al. The Cancer Cell Line Encyclopedia enables predictive modelling of anticancer drug sensitivity. Nature 483, 603-607 (2012).

21. Rhodes, D. R. et al. Oncomine 3.0: genes, pathways, and networks in a collection of 18,000 cancer gene expression profiles. Neoplasia 9, 166-180 (2007).
22. Huang da, W., Sherman, B. T. \& Lempicki, R. A. Systematic and integrative analysis of large gene lists using DAVID bioinformatics resources. Nat. Protoc. 4, 44-57 (2009).

23. Szklarczyk, D. et al. STRINGv10: protein-protein interaction networks, integrated over the tree of life. Nucleic Acids Res. 43, D447-D452 (2015).

24. Sakaguchi, $\mathrm{K}$. et al. Damage-mediated phosphorylation of human p53 threonine 18 through a cascade mediated by a casein 1-like kinase. Effect on Mdm2 binding. J. Biol. Chem. 275, 9278-9283 (2000).

25. Hill, R., Bodzak, E., Blough, M. D. \& Lee, P. W. p53 binding to the p21 promoter is dependent on the nature of DNA damage. Cell Cycle 7, 2535-2543 (2008).

26. Dinkel, H. et al. ELM2016--data update and new functionality of the eukaryotic linear motif resource. Nucleic Acids Res. 44, D294-D300 (2016).

27. Selvarajan, S. \& Shanmughavel, P. A web based database for hypothetical genes in the human genome. Int. J. Comput. Appl. 14, 10-13 (2011).

28. Mitchell, A. et al. The InterPro protein families database: the classification resource after 15 years. Nucleic Acids Res. 43, D213-D221 (2015).

29. Barrett, T. et al. NCBI GEO: archive for functional genomics data sets--update. Nucleic Acids Res. 41, D991-D995 (2013).

30. Brooks, C. L., Li, M., Hu, M., Shi, Y. \& Gu, W. The p53--Mdm2--HAUSP complex is involved in p53 stabilization by HAUSP. Oncogene 26, 7262-7266 (2007).

31. Lee, J. T. \& Gu, W. The multiple levels of regulation by p53 ubiquitination. Cell Death Differ. 17, 86-92 (2010).

32. Epping, M. T. et al. TSPYL5 suppresses p53 levels and function by physical interaction with USP7. Nat. Cell Biol. 13, 102-108 (2011).

33. Marine, J. C. \& Lozano, G. Mdm2-mediated ubiquitylation: p53 and beyond. Cell Death Differ. 17, 93-102 (2010).

34. Rotem, A. et al. Alternative to the soft-agar assay that permits high-throughput drug and genetic screens for cellular transformation. Proc. Natl Acad. Sci. USA 112, 5708-5713 (2015).

35. Choi, E. S., Kim, H., Kim, H. P., Choi, Y. \& Goh, S. H. CD44v8-10 as a potential theranostic biomarker for targeting disseminated cancer cells in advanced gastric cancer. Sci. Rep. 7, 4930 (2017).

36. Untergasser, A. et al. Primer3--new capabilities and interfaces. Nucleic Acids Res. 40, e115 (2012)

37. Laptenko, O., Beckerman, R., Freulich, E. \& Prives, C. p53 binding to nucleosomes within the p21 promoter in vivo leads to nucleosome loss and transcriptional activation. Proc. Natl Acad. Sci. USA 108, 10385-10390 (2011). 